\title{
A cerebrospinal fluid microRNA analysis: Progressive supranuclear palsy
}

\author{
WAKAKO NONAKA ${ }^{1}$, TADAYUKI TAKATA ${ }^{1}$, HISAKAZU IWAMA ${ }^{2}$, SATOSHI KOMATSUBARA $^{3}$, \\ HIDEKI KOBARA $^{4}$, MASAKI KAMADA ${ }^{1}$, KAZUSHI DEGUCHI ${ }^{1}$, TETSUO TOUGE ${ }^{5}$, \\ OSAMU MIYAMOTO ${ }^{6}$, TAKEHIRO NAKAMURA ${ }^{7}$, TOSHIFUMI ITANO ${ }^{1}$ and TSUTOMU MASAKI $^{4}$
}

\author{
${ }^{1}$ Department of Neurology, Faculty of Medicine; ${ }^{2}$ Life Science Research Center; Departments of ${ }^{3}$ Orthopedic Surgery, \\ ${ }^{4}$ Gastroenterology and ${ }^{5}$ Health Sciences, Faculty of Medicine, Kagawa University, Miki-Cho, Kagawa 761-0793; \\ ${ }^{6}$ Department of Medical Engineering, Faculty of Health Science and Technology, Kawasaki University of Medical Welfare, \\ Kurashiki, Okayama 701-0193; ${ }^{7}$ Department of Physiology 2, Kawasaki Medical School, Kurashiki, Okayama 701-0192, Japan
}

Received July 13, 2021; Accepted December 7, 2021

DOI: $10.3892 / \mathrm{mmr} .2022 .12604$

\begin{abstract}
Progressive supranuclear palsy (PSP) is a neurodegenerative tauopathy described as a syndrome of postural instability, supranuclear vertical gaze palsy, dysarthria, dystonic rigidity of the neck and trunk, dementia, and pseudobulbar palsy. The clinical diagnosis of PSP is often difficult because there are no established biomarkers, and diagnosis is currently based on clinical and imaging findings. Furthermore, the etiology and pathogenesis of PSP remain unknown. Dysregulation of microRNAs (miRNAs/miRs) has been reported to serve an important role in neurodegenerative diseases. However, the miRNA profiles of patients with PSP are rarely reported. The present study aimed to examine cerebrospinal fluid miRNAs, which are considered to be more sensitive indicators of changes in the brain, to elucidate the pathophysiology of PSP and to establish specific biomarkers for diagnosis. The present study used a microarray chip containing 2,632 miRNAs to examine cerebrospinal fluid miRNA expression levels in 11 patients with PSP aged 68-82 years. A total of 8 age- and sex-matched controls were also included. A total of 38 miRNAs were significantly upregulated and one miRNA was significantly downregulated in the cerebrospinal fluid of patients with PSP. The patients were divided into two groups based on disease stage (early onset and advanced), and changes in miRNA expression were examined. The miRNAs that were most significantly upregulated or downregulated in the early onset group were miR-204-3p, miR-873-3p and miR-6840-5p. The target genes of these miRNAs were associated with molecules related to the ubiquitin-proteasome
\end{abstract}

Correspondence to: Dr Wakako Nonaka, Department of Neurology, Faculty of Medicine, Kagawa University, 1750-1 Ikenobe, Miki-Cho, Kita-Gun, Kagawa 761-0793, Japan

E-mail: nonaka.wakako@kagawa-u.ac.jp

Key words: progressive supranuclear palsy, microRNA, cerebrospinal fluid, humans, Parkinson's and related diseases system and autophagy pathway. Furthermore, these miRNAs were found to target genes that have been reported to have epigenetic changes following an epigenome-wide association study of brain tissues of patients with PSP. This suggested that these miRNAs and genes may have some involvement in the pathogenesis of PSP. However, the sample size of the present study was small; therefore, a greater number of patients with PSP should be examined in future studies.

\section{Introduction}

Neurodegenerative diseases are generally progressive and refractory because they involve irreversible degeneration of brain neurons and many remain incurable. Treatments to slow progression after disease onset do not compensate for neural tissues that have already been lost and do not result in a retrograde recovery of symptoms. Therefore, it is important to implement therapeutic interventions and risk management as early as possible in order to delay disease onset and progression and to extend healthy life expectancy (1). However, this is difficult because diagnosis is based on the detection of clinical symptoms and neuroimaging abnormalities, which appear relatively late in the disease course. Nonetheless, molecular responses to genetic and environmental disorders begin much earlier, and non-coding RNA networks have been found to be involved in these cellular regulatory mechanisms. MicroRNAs (miRNAs) are small non-coding RNAs of approximately 20-25 nucleotides, and more than 2,000 different miRNAs have been suggested to exist in humans (2). MiRNAs repress the expression of various genes in vivo and exhibit unique disease-specific expression patterns. In addition, miRNAs are stable in body fluids such as blood, urine, and saliva, which are easy to collect from living organisms, and can be detected with high sensitivity (3). Thus, profiling the miRNA expression patterns may facilitate the detection of diseases at a preliminary stage. Indirect evaluation of pathological processes is particularly important in neurological diseases, where direct access to tissues for molecular analysis is not possible. Living neurons and other cells of the central nervous system (CNS) 
secrete miRNAs wrapped in exosomes, microvesicles, and lipoprotein complexes (4). Interactions between external stimuli and pathological processes in the brain have been reported to be reflected in peripheral tissues, and their use as potential diagnostic markers is of interest. Cerebrospinal fluid (CSF) has also been shown to contain circulating miRNAs. As the CSF is in direct contact with the brain, it is said to reflect changes in the brain and may be suitable as a biomarker (5). Recently, specific miRNAs in the CSF have been reported in Alzheimer's disease (6) and Parkinson's disease (PD) (7) and are expected to be potential diagnostic markers and therapeutic targets.

Progressive supranuclear palsy (PSP) is a progressive neurodegenerative tauopathy characterized by postural instability with falls, vertical supranuclear gaze palsy, parkinsonism with poor levodopa response, pseudobulbar palsy, and frontal release signs (8). The mean age of onset of PSP is approximately 65 years and the condition leads to death within approximately 6-9 years after symptom onset $(9,10)$. The factors involved in its pathogenesis are still unknown, and no fundamental treatment has been established. In addition, the diagnosis of PSP is currently dependent on clinical and imaging findings, which makes early diagnosis difficult.

It is difficult to differentiate PSP from PD and the other Parkinsonian syndromes such as multiple system atrophy (MSA) and corticobasal degeneration (CBD), especially in the early stages. The differences in the Parkinsonian syndrome, which has similar clinical symptoms to PSP, are presented in Table SI. To improve the accuracy of the clinical diagnosis of Parkinsonian syndrome, CSF markers have been explored (11). Therefore, we focused on miRNAs as one candidate that could lead to early diagnosis of PSP.

At present, there are very few reports of miRNA expression profiling in PSP. It has been reported that miR-132 (12), miR-147a, and miR-518e (13) are expressed in the brain tissues of patients with PSP, but these miRNAs are rarely reported in biofluids. We herein aimed to examine CSF miRNAs, which are thought to be a sensitive indicator of changes in the brain, to elucidate the pathophysiology of PSP and establish specific biomarkers for diagnosis.

\section{Materials and methods}

Participants. Eleven patients with PSP (nine men and two women; median age, 76 years; range, 68-82 years) and eight age- and sex-matched controls (seven men and one woman; median age, 75.5 years; range, 63-89 years) were recruited for the present study between January 2017 and December 2018 from the Department of Neurology, Kagawa University Hospital. All participants in this study were Japanese. We enrolled patients fulfilling the Movement Disorder Society (MDS) clinical diagnostic criteria for PSP (MDS-PSP criteria) (14) and for probable PSP, Richardson's syndrome (PSP-RS) phenotype according to their disease history and clinical information including neuroimaging findings. All patients had sporadic PSP. Patient background data (age, sex, disease duration, comorbidities, and clinical parameters) at the time of sample collection were retrospectively obtained from medical records. Clinical parameters included scores on the PSP Rating Scale (PSPRS) (15), Mini-Mental State Examination (MMSE) (16), Frontal Assessment Battery (FAB) (17), and Montreal Cognitive Assessment (MoCA) (18).
We assessed brain magnetic resonance imaging scans for findings suggestive of PSP. Furthermore, we applied the magnetic resonance parkinsonism index (MRPI) (19) and MRPI 2.0 (20). The threshold for the MRPI and MRPI 2.0 was $>13.88$ and $>2.50$, respectively (20). Patients with PSP were divided into two subgroups: The 'early disease stage' subgroup included those who underwent neurological evaluation within 2 years of symptom onset and the 'advanced disease stage' subgroup included patients who underwent evaluation $>2$ years after symptom onset. Patients with concurrent malignant tumors, psychiatric disorders, collagen diseases, endocrine diseases, or infections were excluded because these conditions may alter the expression profile of some miRNAs. Owing to the above-mentioned reason, participants in the control group with concurrent malignant tumors, psychiatric disorders, collagen diseases, endocrine diseases, or infections were also excluded. In addition, participants with neurological diseases such as dementia and parkinsonism were excluded. The study was approved by the Medical Ethics Committee of Kagawa University (Kagawa, Japan) (Ethics approval Heisei 22-002). Participants and controls received a full explanation of the study purpose and protocol and provided written informed consent before inclusion in the study.

CSF miRNA expression profiling. CSF samples were obtained during lumbar puncture between the L3 and L5 vertebrae with the participant in a lateral supine position. Lumbar punctures were performed between 9 and $12 \mathrm{am}$, using a sterile technique and a 21-23G spinal needle in accordance with local guidelines. At least $4 \mathrm{ml}$ of CSF was collected from each participant. CSF was collected in polypropylene tubes and cytologically controlled to be free of erythrocytes. The sample was centrifuged $(2,000 \mathrm{x} \mathrm{g}$, $4^{\circ} \mathrm{C}, 10 \mathrm{~min}$ ), frozen immediately, and stored at $-80^{\circ} \mathrm{C}$ until use. The CSF was at no time thawed/refrozen. Total RNA was extracted from $900 \mu \mathrm{l}$ of frozen CSF using a 3D-Gene ${ }^{\circledR}$ RNA extraction reagent (Toray) according to the manufacturer's specifications. The BioAnalyzer 2100 system (Agilent Technologies, Inc.) was used to determine the RNA integrity number (RIN) for RNA quality assessment. No difference in total RNA concentration or RIN was observed between the PSP group and controls. After labeling with a 3D-Gene miRNA labeling kit (Toray), the extracted total RNA was hybridized onto the 3D-Gene ${ }^{\circledR}$ Human miRNA oligo chip Version 22.0 (Toray) with 2,632 fluorescent probes. Hybridization signals were obtained using a 3D-Gene 3000 miRNA microarray scanner (Toray). The raw data of each detected signal were normalized by the global normalization method, subtracting the mean intensity of the background signal in each microarray, and the median of the detected signal intensity was adjusted to 25. Microarray data have been deposited in NCBI's Gene Expression Omnibus (21) and are accessible through GEO Series accession number GSE186921 (https:/www.ncbi.nlm.nih.gov/geo/query/acc. cgi?acc $=$ GSE186921).

Heatmap. Hierarchical clustering was performed on the PSP and control groups, using a subset of miRNAs determined to be significantly differentially expressed between the two groups. The Ward agglomerative method was used to cluster both the samples and miRNAs. The heatmap was color-coded according to the expression levels. The center level of the color code was set as the median value over all of the values 
Table I. General and clinical characteristics of the participants.

\begin{tabular}{|c|c|c|c|c|}
\hline Variable & Control $(n=8)$ & PSP early stage $(n=5)$ & PSP advanced stage $(n=6)$ & P-value \\
\hline \multicolumn{5}{|l|}{ Sex, $\mathrm{n}$} \\
\hline Male & 7 & 3 & 6 & $0.316^{\mathrm{a}}$ \\
\hline Female & 1 & 2 & 0 & \\
\hline Median (IQR) age at test, years & $75.5(64.8-79.0)$ & $75.0(69.0-76.0)$ & $76.5(70.8-79.8)$ & $0.516^{\mathrm{b}}$ \\
\hline Median (IQR) disease duration, years & ND & $2(2)$ & $5(4-6)$ & $0.005^{\mathrm{c}}$ \\
\hline Median (IQR) PSPRS score,/100 & ND & $26.0(24.5-31.0)$ & $58.0(51.8-62.3)$ & $0.008^{\mathrm{c}}$ \\
\hline Median (IQR) MMSE score $/ 30$ & ND & $26.0(25.0-28.0)$ & $19.0(11.5-20.3)$ & $0.008^{\mathrm{c}}$ \\
\hline Median (IQR) FAB score,/18 & ND & $12.0(10.5-13.5)$ & $6.0(5.0-9.3)$ & $0.042^{\mathrm{c}}$ \\
\hline Median (IQR) MoCA score, $/ 30$ & ND & $21.0(19.0-23.0)$ & $8.5(5.0-12.8)$ & $0.008^{\mathrm{c}}$ \\
\hline Median (IQR) MRPI & ND & $30.6(23.0-31.2)$ & $42.6(37.9-48.1)$ & $0.014^{\mathrm{c}}$ \\
\hline Median (IQR) MRPI 2.0 & ND & $6.8(5.0-7.9)$ & $10.9(9.7-14.2)$ & $0.008^{\mathrm{c}}$ \\
\hline
\end{tabular}

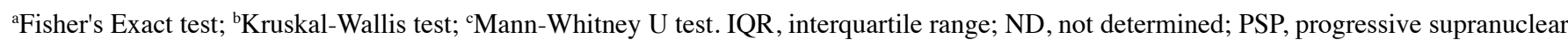
palsy; PSPRS, progressive supranuclear palsy rating scale; MMSE, Mini-Mental State Examination; FAB, Frontal Assessment Battery; MoCA, Montreal Cognitive Assessment; MRPI, Magnetic Resonance Parkinsonism Index.

used in the heatmap. Gray color represented mean values, red indicated an increase, and blue represented a decrease in expression. The heatmap was created using JMP ${ }^{\circledR}$ Pro version 15.1.0 (SAS Institute Inc.).

miRNA target gene bioinformatics analysis. Using in silico analysis, the target genes of miRNAs were predicted using the miRDB (http://mirdb.org/miRDB/), Targetscan 7.2 (http://www.targetscan.org/), and mirTarBase version 8.0 (http://mirtarbase.mbc.nctu.edu.tw/) databases. To enhance the reliability of the bioinformatics analysis, overlapping target genes were identified in this analysis.

Statistical analysis. For comparisons of clinical characteristics between the groups, the Mann-Whitney U test was used to compare all variables except for sex and age. The Fisher's exact test and Kruskal-Wallis test were used to compare sex and age, respectively. The median (interquartile range) values for each parameter are presented. Statistical analysis of miRNA expression levels in each group was conducted using a non-parametric Mann-Whitney U test and the Kruskal-Wallis test as appropriate. Benjamini-Hochberg procedures for adjusting the false discovery rate (FDR) in multiple comparisons were also applied. Multiple comparisons of continuous variables among multiple groups were performed using the Kruskal-Wallis test, followed by the Steel-Dwass post-hoc test. P-values of $<0.05$ were regarded as statistically significant. Statistical analyses were performed using JMP ${ }^{\circledR}$ Pro version 15.1.0 (SAS Institute Inc.).

\section{Results}

Clinical characteristics of participants. The clinical characteristics are presented in Table I. There were no significant differences in the sex ratio and age between the PSP and control groups. The PSPRS scores and disease severity were significantly higher in the advanced stage group than in the early stage group. The scores on the cognitive assessment

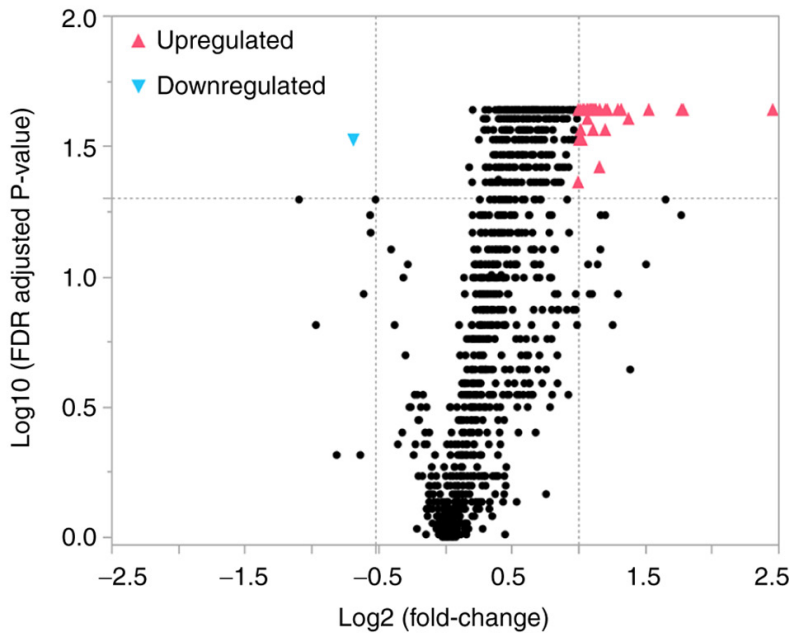

Figure 1. Volcano plot of the miRNAs isolated from the cerebrospinal fluid of patients with progressive supranuclear palsy and controls. The volcano plot illustrates the relationship between the fold change and significance of the difference between the two groups. The $y$-axis depicts the negative $\log _{10}$ of FDR-adjusted P-values (the horizontal line at 1.3 corresponds to a P-value of 0.05 , a higher value indicates greater significance) and the $\mathrm{X}$-axis is the difference in expression between the two experimental groups as $\log _{2}$ fold changes (the vertical lines indicate that miRNAs are either upregulated or downregulated above a fold change of 2 and 0.7 , respectively). The blue color indicates significantly downregulated miRNAs and the red color indicates significantly upregulated miRNAs. FDR, false discovery rate; miRNA, microRNA.

scale, MMSE, MoCA, and FAB were significantly lower in the advanced stage group than in the early stage group. There was also a significant difference in the imaging scale scores between the early and advanced stage groups.

CSF miRNA expression profile. Of the 2,632 miRNAs detectable on the 3D-Gene ${ }^{\circledR}$ Human miRNA OligoChip (Version 22.0), 1,104 were detected in all CSF samples from both the PSP and control groups. The volcano plot (Fig. 1) shows the relationship between the fold change and significance of 1,104 detectable miRNAs between the two groups. 
Table II. Statistical results, chromosomal locations and known associated diseases of miRNAs which were significantly altered in patients with progressive supranuclear palsy.

\begin{tabular}{|c|c|c|c|c|c|}
\hline MiRNA & Fold change & $\mathrm{P}$-value & $\begin{array}{l}\text { FDR adjusted } \\
\text { P-value }\end{array}$ & $\begin{array}{l}\text { Chromosomal } \\
\text { localization }\end{array}$ & miRNA-disease association \\
\hline \multicolumn{6}{|l|}{ Upregulated } \\
\hline hsa-miR-204-3p & 5.50 & 0.0003 & 0.0230 & $9 q 21.12$ & $\begin{array}{l}\text { Retinal dystrophy and iris coloboma with or } \\
\text { without cataract }(22)\end{array}$ \\
\hline hsa-miR-4476 & 3.46 & 0.0034 & 0.0230 & $9 \mathrm{p} 13.2$ & \\
\hline hsa-miR-6132 & 3.42 & 0.0015 & 0.0230 & $7 q 31.2$ & \\
\hline hsa-miR-4638-5p & 2.89 & 0.0003 & 0.0230 & $5 q 35.3$ & \\
\hline hsa-miR-7110-5p & 2.60 & 0.0044 & 0.0249 & $3 q 21.1$ & \\
\hline hsa-miR-3679-5p & 2.50 & 0.0015 & 0.0230 & $2 q 21.2$ & \\
\hline hsa-miR-1236-5p & 2.46 & 0.0011 & 0.0230 & $6 \mathrm{p} 21.33$ & \\
\hline hsa-miR-6867-3p & 2.33 & 0.0026 & 0.0230 & $17 q 21.1$ & \\
\hline hsa-miR-6761-3p & 2.31 & 0.0011 & 0.0230 & $12 \mathrm{q} 24.12$ & \\
\hline hsa-miR-423-5p & 2.30 & 0.0057 & 0.0275 & $17 q 11.2$ & \\
\hline hsa-miR-7111-3p & 2.25 & 0.0020 & 0.0230 & $6 \mathrm{p} 21.31$ & \\
\hline hsa-miR-3156-3p & 2.24 & 0.0015 & 0.0230 & $21 \mathrm{q} 11.2$ & \\
\hline hsa-miR-12114 & 2.24 & 0.0118 & 0.0382 & $22 q 13.33$ & \\
\hline hsa-miR-6889-5p & 2.20 & 0.0026 & 0.0230 & $22 q 13.2$ & \\
\hline hsa-miR-6740-3p & 2.20 & 0.0011 & 0.0230 & $1 \mathrm{q} 32.1$ & \\
\hline hsa-miR-885-5p & 2.18 & 0.0020 & 0.0230 & $3 \mathrm{p} 25.3$ & \\
\hline hsa-miR-6894-3p & 2.18 & 0.0020 & 0.0230 & Xp11.22 & \\
\hline hsa-miR-487b-5p & 2.16 & 0.0015 & 0.0230 & $14 q 32.31$ & Neuroblastomas (23) \\
\hline hsa-miR-6820-5p & 2.16 & 0.0057 & 0.0275 & $22 q 13.1$ & \\
\hline hsa-miR-873-3p & 2.15 & 0.0008 & 0.0230 & $9 \mathrm{p} 21.1$ & Multiple sclerosis (24) \\
\hline hsa-miR-7109-3p & 2.14 & 0.0026 & 0.0230 & $22 \mathrm{q} 12.2$ & \\
\hline hsa-miR-5193 & 2.12 & 0.0015 & 0.0230 & $3 \mathrm{p} 21.31$ & \\
\hline hsa-miR-4648 & 2.10 & 0.0034 & 0.0230 & $7 \mathrm{p} 22.3$ & \\
\hline hsa-miR-10398-5p & 2.10 & 0.0044 & 0.0249 & $6 \mathrm{p} 21.1$ & \\
\hline hsa-miR-1825 & 2.10 & 0.0015 & 0.0230 & $20 q 11.21$ & \\
\hline hsa-miR-6870-5p & 2.10 & 0.0026 & 0.0230 & $20 \mathrm{p} 12.2$ & \\
\hline hsa-miR-6825-5p & 2.07 & 0.0034 & 0.0230 & $3 q 21.3$ & \\
\hline hsa-miR-4700-3p & 2.07 & 0.0020 & 0.0230 & $12 \mathrm{q} 24.31$ & \\
\hline hsa-miR-3622a-3p & 2.05 & 0.0011 & 0.0230 & $8 \mathrm{p} 21.1$ & \\
\hline hsa-miR-5001-5p & 2.04 & 0.0073 & 0.0300 & $2 q 37.1$ & \\
\hline hsa-miR-6510-5p & 2.04 & 0.0073 & 0.0300 & $17 \mathrm{q} 21.2$ & \\
\hline hsa-miR-4505 & 2.04 & 0.0073 & 0.0300 & $14 q 24.3$ & \\
\hline hsa-miR-4665-5p & 2.03 & 0.0057 & 0.0275 & $9 \mathrm{p} 24.1$ & \\
\hline hsa-miR-8485 & 2.02 & 0.0008 & 0.0230 & $2 \mathrm{p} 16.3$ & \\
\hline hsa-miR-7110-3p & 2.01 & 0.0073 & 0.0300 & $3 q 21.1$ & \\
\hline hsa-miR-6862-3p & 2.00 & 0.0015 & 0.0230 & $16 \mathrm{p} 12.1$ & \\
\hline hsa-miR-6886-3p & 2.00 & 0.0034 & 0.0230 & $19 \mathrm{p} 13.2$ & \\
\hline hsa-miR-328-5p & 2.00 & 0.0149 & 0.0436 & $16 q 22.1$ & Myelogenous leukemia (25) \\
\hline Downregulated & & & & & \\
\hline hsa-miR-6840-5p & 0.62 & 0.0073 & 0.0300 & $7 q 22.1$ & \\
\hline
\end{tabular}

Significant differential miRNA expression was determined by a fold change ratio of $>2$ or $<0.7, \mathrm{P}<0.05$, and FDR-adjusted $\mathrm{P}<0.05$. Most of the miRNAs that exhibited changes in the present study were relatively newly discovered, and their target genes and functions were not fully understood. FDR, false discovery rate; miRNA/miR, microRNA.

Thirty-eight miRNAs had significantly higher (upregulated) and one miRNA had significantly lower (downregulated) normalized values in the PSP group than in the control group, as assessed by a fold change ratio of $>2$ or $<0.7$ and an 


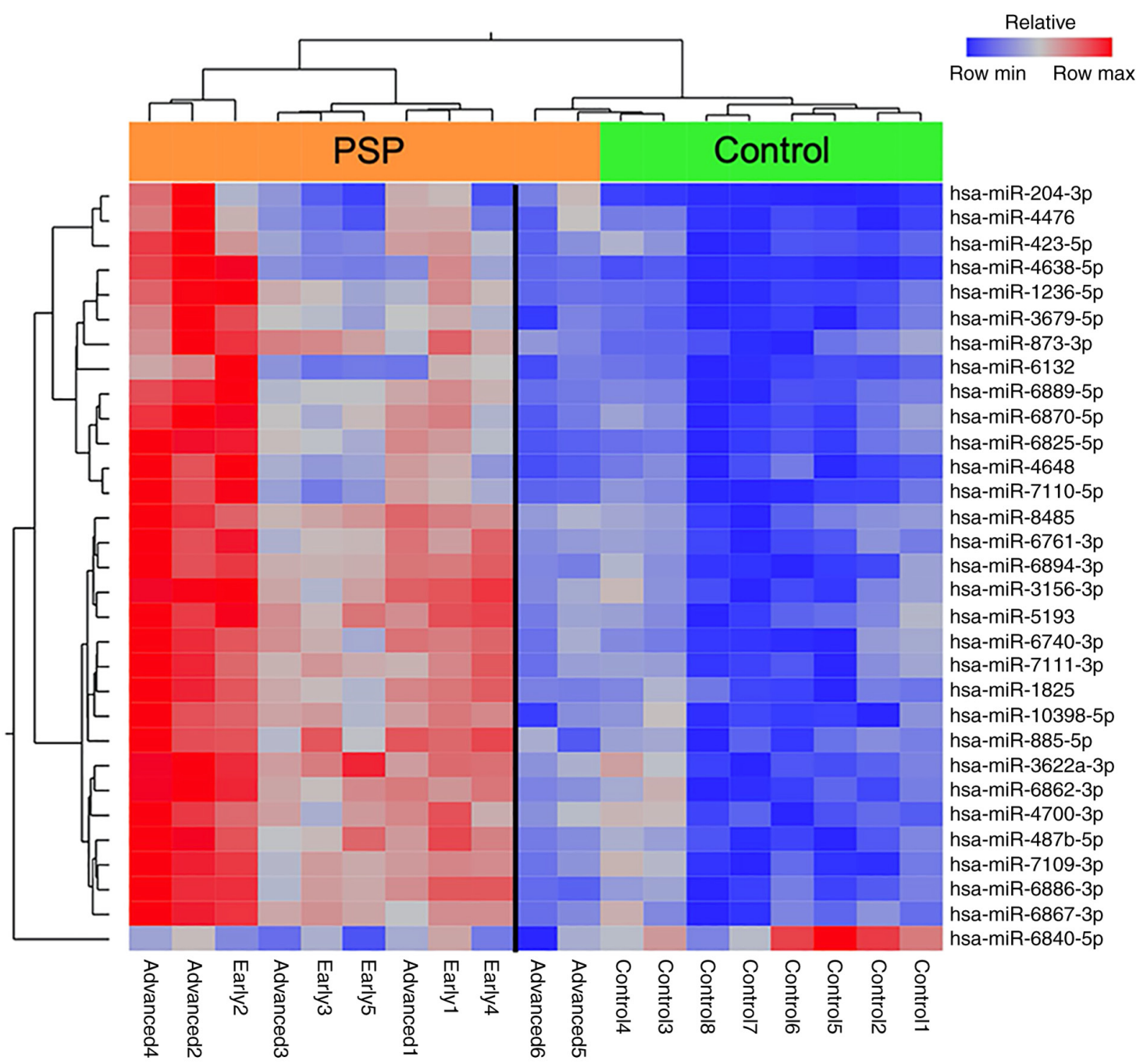

Figure 2. Heatmap representing the hierarchical clustering of aberrantly expressed miRNAs. Samples are arranged in columns and miRNAs in rows. The miRNA clustering tree is presented on the left and the sample clustering tree is present at the top of each heat map. The heat map depicts the relative expression intensity for each miRNA: Red cells indicate high levels of expression and blue cells indicate levels of low expression. The orange bar represents the PSP group and the green bar represents the control group. miRNA/miR, microRNA; PSP, progressive supranuclear palsy.

FDR-adjusted P-value of $<0.05$. These miRNAs are indicated in Table II. The chromosomal locations of the miRNAs and known disease associated with the miRNAs (22-25) are also presented in Table II.

The extent to which the upper 30 miRNAs with high expression levels and one with low expression levels in patients with PSP separated from controls was further explored by hierarchical clustering, which revealed a clear partition between the PSP and control groups when the two patients with the longest disease duration were excluded from the analysis (Fig. 2).

Differences between the early and advanced stage groups. We selected up- and downregulated miRNAs with the most significant difference in the early stage group. The top two upregulated miRNAs and the top downregulated miRNA that were selected are presented in Table III and in Fig. 3. The expression level of hsa-miR-204-3p in the CSF was higher than that of other miRNAs. Hsa-miR-204-3p expression levels were significantly higher in the early stage group than in the control group $(\mathrm{P}=0.0043)$. Although the difference was not significant, hsa-miR-204-3p was also increased in the advanced stage group compared to in the early stage group $(\mathrm{P}=0.0552)$ (Fig. 3A). Hsa-miR-873-3p was also significantly elevated in the early stage group $(\mathrm{P}=0.0043)$ and remained high in the advanced stage group (Fig. 3B). In contrast, hsa-miR-6840-5p was downregulated in the early stage group compared to controls $(\mathrm{P}=0.0338)$ but did not significantly differ between the early and advanced stage groups (Fig. 3C).

\section{Discussion}

In the present study, we investigated changes in the expression of a wide range of miRNAs via microarray analysis in samples from patients with PSP and healthy controls. In previous studies, the analysis of miRNA expression profiles among patients with PSP showed that miR-132 is specifically downregulated in the brain tissue (12). miR-147a and miR-518e were also significantly upregulated in the forebrain tissue of patients with PSP (13). In this study, miR-132 was detected at low levels in the CSF of all participants. However, miR-147a was not detected in the control group but was detected in some patients with PSP $(n=8 / 11,73 \%)$. miR-518e-5p expression was 
Table III. Upregulated and downregulated miRNAs in the subgroups of patients with progressive supranuclear palsy.

\begin{tabular}{lcc}
\hline MiRNA & P-value (Kruskal-Wallis) & Post hoc (Steel-Dwass) \\
\hline Upregulated & & 0.0120 \\
hsa-miR-204-3p & 0.0043 & 0.0068 \\
$\quad$ Control vs. PSP early & 0.0024 & 0.1338 \\
Control vs. PSP advanced & 0.0552 & 0.0120 \\
PSP early vs. PSP advanced & & 0.0222 \\
hsa-miR-873-3p & 0.0043 & 0.6896 \\
Control vs. PSP early & 0.0081 & 0.4113 \\
Control vs. PSP advanced & & 0.0853 \\
PSP early vs. PSP advanced & & 0.0617 \\
Downregulated & 0.0338 & 0.9954 \\
hsa-miR-6840-5p & 0.0239 & 0.9273 \\
Control vs. PSP early & & \\
Control vs. PSP advanced & & \\
PSP early vs. PSP advanced & & \\
\hline
\end{tabular}

miRNA/miR, microRNA; PSP, progressive supranuclear palsy.
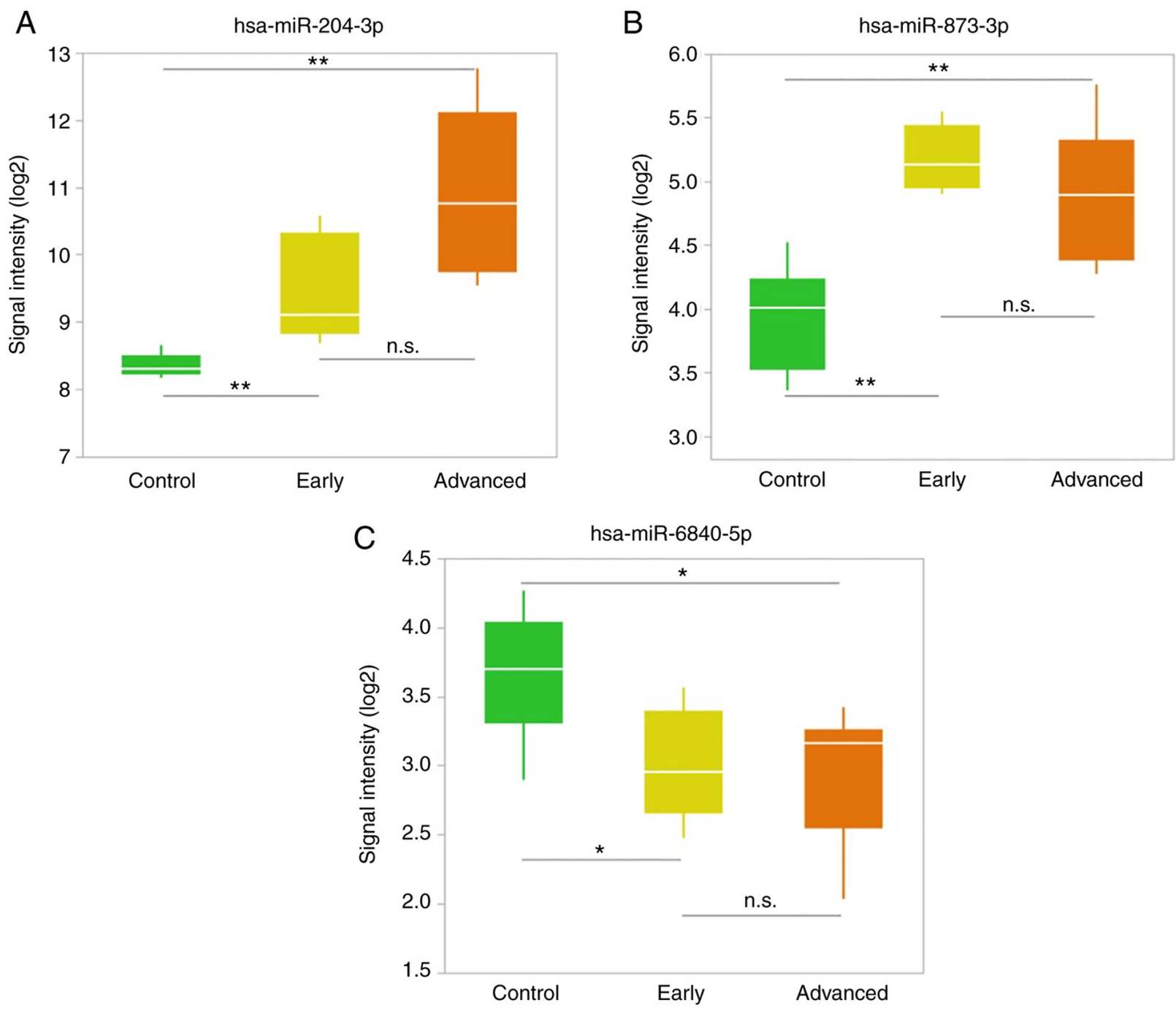

Figure 3. Upregulated and downregulated miRNAs in the subgroups of patients with progressive supranuclear palsy. (A) Upregulated miRNA that was progressively elevated from early to advanced stages of disease. (B) Upregulated miRNA that was elevated from an early stage of illness and maintained that trend into the advanced stage. (C) Downregulated miRNA in the early stage disease group. ${ }^{* *} \mathrm{P}<0.01,{ }^{*} \mathrm{P}<0.05$. miRNA/miR, microRNA; n.s., not significant. 
Table IV. Prediction of the genes targeted by the three miRNAs.

\begin{tabular}{ll} 
miRNA & \multicolumn{1}{c}{ Target genes } \\
\hline miR-204-3p & UNG, NUP50, TBL1XR1, PIK3C2B, CNNM4, NFASC, KRAS, APOLD1, METTL1, ACAP2, \\
& MOB3A, TGFBR1, SEPT6, XKR7, AJAP1 \\
miR-873-3p & MIDN, ZFX, USP36, CC2D2A, CD40LG, MXRA7 \\
miR-6840-5p & ENPP1, PFKFB3, ICAM5, IL1RAPL1, FBXL18, PITX1, MGRN1, PPP1R14B
\end{tabular}

miRNA/miR, microRNA.

significantly downregulated in patients with PSP relative to controls (fold change ratio $=0.76, \mathrm{P}=0.03$, data not shown), and there was no significant difference in the expression between the early and advanced stage groups. The profile of circulating miRNAs in the CSF in our study differed slightly from that of miRNAs in the brain tissue reported in previous studies, indicating that not all altered miRNAs in the tissue are released as circulating miRNAs.

In addition, miRNAs with altered expression levels were divided into early and advanced disease stages to understand the pathophysiology of the disease process by following the changes in miRNAs over the course of disease. We found that in the early stages of the disease, hsa-miR-204-3p and hsa-miR-873-3p expression levels were significantly elevated in the PSP group compared to those in the control group, and hsa-miR-6840-5p expression was significantly lower in the PSP group than in the control group. Genes that change from an early stage of PSP and show similar changes in the advanced stage were thought to be more involved in the pathogenesis of PSP. Genes that show a gradual increase from early to advanced stages may reflect the process of neurodegeneration.

In silico analysis was performed for each miRNA, and the significance of miRNA expression changes was examined from the predicted target genes. The target genes were predicted using multiple databases, particularly for the miRNAs that were significantly altered in the early stage group (miR-204-3p, miR-873-3p, and miR-6840-5p). To further enhance the reliability of the bioinformatics analysis, overlapping target genes were identified (Table IV).

miR-204-3p was abundantly expressed in the PSP and control groups, and the expression level showed a small dispersion and equivalency in the control group. Further, the expression level in the PSP group increased from the early to advanced stages of disease. miR-204-3p upregulation in the CSF has not been reported previously, while miR-204-5p expression level was demonstrated to be low in the CSF of patients with frontotemporal dementia (26) or MSA (27). In this study, miR-204-3p was significantly upregulated in the CSF of patients with PSP. Activating transcription factor 2 (ATF2) (28), regulator of G-protein signaling 5 (RGS5) (29), protein phosphatase, $\mathrm{Mg} 2+/ \mathrm{Mn} 2+$ dependent $1 \mathrm{~K}$ (30), and $\mathrm{K}$ homology domain-containing, RNA-binding, signal transduction-associated protein 1 (KHDRBS1) (31) are confirmed targets of miR-204. miR-204 has been reported to be involved in the process of autophagy (32). ATF2 is involved in the regulation of cell proliferation, apoptosis, or autophagy of several cancers, such as glioblastoma (28), non-small cell lung cancer (33), and cervical cancer (34). KHDRBS1 is also known as Sam68 or p62 and is widely known as the central regulator between the ubiquitin-proteasome system and autophagy (35). The predicted target genes of miR-204-3p are listed in Table IV. Among them, miR-204-3p was predicted to target adherens junctions associated protein 1 (AJAPl) (36). Interestingly, $A J A P 1$ was found to be hypermethylated in patients with PSP in an epigenome-wide association study (EWAS) (37). These data suggest that miR-204-3p may be involved in the pathogenesis of PSP.

miR-873-3p is reportedly downregulated in the CSF of patients with $\mathrm{PD}$, but upregulated miR-873-3p expression in the CSF of patients with other neurological disorders has not been reported previously (7). In the present analysis, miR-873-3p was significantly elevated in the early stage group, suggesting that miR-873-3p may be useful in differentiating PSP and $\mathrm{PD}$, which can be difficult to distinguish in the early stages. miR-873-3p was predicted to target midbrain nucleolar protein (midnolin, MIDN) (38-40). MIDN has been confirmed as a genetic risk factor for PD (41) and is suggested to be a novel regulator of parkin expression via the promotion of parkin E3 ubiquitin ligase expression (42). In this regard, hsa-miR-873-3p may be involved in the differences in the pathogenesis of PSP and PD.

In this study, miR-6840-5p expression was downregulated in patients with PSP. As shown in Table IV, miR-6840-5p was predicted to target genes such as the gene encoding F-Box and leucine-rich repeat protein 18 (FBXL18) (43) and Mahogunin ring finger-1 (MGRN1) $(43,44)$, as previously reported. Interestingly, $F B X L 18$ was found to be hypomethylated in patients with PSP in an EWAS (37). FBXL18 has been reported to bind directly to leucine-rich repeat kinase 2 (LRRK2), regulate LRRK2 stability, and control LRRK2-mediated toxicity (45). In contrast, $M G R N 1$, an E3 ubiquitin ligase of the really interesting new gene finger family, is a crucial component in autophagy and the ubiquitin-proteasome system and is involved in abnormal protein degradation and quality control. Previous findings have indicated that dysfunctional MGRN1 can cause neurodegeneration and mitochondrial dysfunction in mouse brains (46). In summary, we found that many of the genes targeted by miRNAs with significantly altered expression levels in this study were involved in the ubiquitin-proteasome system and autophagy pathway.

Recently, Ramaswamy's group proposed a role for miRNAs in the expression of key pathological features of Parkinsonian movement disorders. Disruption of protein 
homeostasis mechanisms such as autophagy due to miRNA dysregulation has been suggested to be involved in neurodegeneration. According to them, miRNAs, such as miR-204, play a role in the regulation of the autophagy pathway (47). Interestingly, the expression of miRNAs, which may be involved in the autophagy pathway, was also found to be variable in this study.

There were a number of limitations to this study. The miRNA expression levels could not be validated, and individual miRNA expression levels should be determined using reverse transcription quantitative polymerase chain reaction in the future. In addition, it is necessary to investigate the expression of these miRNAs in serum, which is very accessible and will make it easier to use as a biomarker. Furthermore, the sample size was small as PSP is a rare disease; moreover, this was a single-center study and patients with concurrent malignant tumors, psychiatric diseases, collagen diseases, endocrine diseases, or infectious diseases were excluded. In addition, the collection of CSF samples is more invasive than that of other body fluid samples such as blood; therefore, the number of participants was limited, and the study was conducted on a small sample size. There is only one previous report of profiling data of CSF miRNAs in PSP patients. An miRNA panel including 372 miRNAs was used (21). In this study, we used a microarray chip containing 2632 miRNAs to perform a comprehensive analysis that included many miRNAs that had not been analyzed in previous studies. Therefore, most of the miRNAs that showed changes in this study were relatively newly discovered, and their target genes and functions were not fully understood. Thus, there was a limited number of miRNAs for which in silico analysis was possible. Although this study is based on data from a small sample size, we believe that the results of this analysis are significant in terms of their novelty and originality. In future studies, we would like to examine miRNA expression in a greater number of patients with PSP.

In conclusion, we identified miRNAs that were significantly altered in the CSF of patients with PSP using microarrays. Among them, some miRNAs (hsa-miR-204-3p, 873-3p, and 6840-5p) were significantly altered from an early stage of the disease. We performed bioinformatics analysis of the genes associated with these miRNAs and found that they are involved in processes such as autophagy and the ubiquitin-proteasome system, which are relevant to cellular quality control. Interestingly, we found altered expression of miRNAs targeting genes that have been reported to show epigenetic changes following EWAS of DNA from brain tissues of PSP patients. This suggests that these miRNAs and genes may have some involvement in the pathogenesis of PSP. In future studies, we will analyze how miRNAs interact with such molecules and how they contribute to the pathogenesis of disease.

\section{Acknowledgements}

Not applicable.

\section{Funding}

No funding was received.

\section{Availability of data and materials}

The microarray datasets generated and/or analyzed during the current study are available in the NCBI Gene Expression Omnibus repository (https://www.ncbi.nlm.nih. gov/geo/query/acc.cgi?acc=GSE186921).

\section{Authors' contributions}

WN, TTa, TI and TM conceived the study. WN, TTa, HI, TI and TM curated data. WN, HI and TI performed formal analysis. KD and TTo conceived the study and curated data. WN, SK, MK and HK performed the experiments. WN, HI, $\mathrm{OM}$ and TN developed the methodology. KD, TTo, TI and TM were involved in project administration. SK, HK, MK, KD, TTo and TM provided resources. HK, KD, TTo, OM, TN and TM supervised the study. WN, TTa, KD, TTo, OM, TN and TI were involved in validation. WN and $\mathrm{HI}$ were involved in visualization. WN and TI wrote the original draft. TTa, HI, KD, TTo, OM, TN, TI and TM reviewed and edited the manuscript. All authors have read and approved the final manuscript. TTa and TI confirm the authenticity of all the raw data.

\section{Ethics approval and consent to participate}

The procedures were approved by the ethics committee of Kagawa University Faculty of Medicine (Miki-cho, Kita-gun, Kagawa, Japan). All patients and/or their relatives provided written informed consent. All clinical investigations have been conducted according to the principles expressed in the Declaration of Helsinki.

\section{Patient consent for publication}

All patients and/or their relatives provided written informed consent.

\section{Competing interests}

The authors declare that they have no competing interests.

\section{References}

1. Agrawal $\mathrm{M}$ and Biswas A: Molecular diagnostics of neurodegenerative disorders. Front Mol Biosci 2: 54, 2015

2. Lee RC, Feinbaum RL and Ambrost V: The C. elegans heterochronic gene lin-4 encodes small RNAs with antisense complementarity to lin-14. Cell 75: 843-854, 1993.

3. Quinn JF, Patel T, Wong D, Das S, Freedman JE, Laurant LC, Carter BS, Hochberg F, van Keuren-Jensen K, Huentelmann M, et al: Extracellular RNAs: Development as biomarkers of human disease. J Extracell Vesicles 4: 27495, 2015.

4. Rao P, Benito E and Fischer A: MicroRNAs as biomarkers for CNS disease. Front Mol Neurosci 6: 39, 2013.

5. Roser AE, Caldi Gomes L, Schünemann J, Maass F and Lingor P: Circulating miRNAs as diagnostic biomarkers for Parkinson's disease. Front Neurosci 12: 625, 2018.

6. Denk J, Boelmans K, Siegismund C, Lassner D, Arlt S and Jahn H: MicroRNA profiling of CSF reveals potential biomarkers to detect Alzheimer's disease. PLoS One 10: e0126423, 2015.

7. Burgos K, Malenica I, Metpally R, Courtright A, Rakela B, Beach T, Shill H, Adler C, Sabbagh M, Villa S, et al: Profiles of extracellular miRNA in cerebrospinal fluid and serum from patients with Alzheimer's and Parkinson's diseases correlate with disease status and features of pathology. PLoS One 9: e94839, 2014. 
8. Steele JC, Richardson JC and Olszewski J: Progressive Supranuclear palsy: A heterogeneous degeneration involving the brainstem, basal ganglia and cerebellum with vertical gaze and pseudobulbar palsy, nuchal dystonia and dementia. Arch Neurol 10: 333-359, 1964

9. Coyle-Gilchrist IT, Dick KM, Patterson K, Vázquez Rodríquez P, Wehmann E, Wilcox A, Lansdall CJ, Dawson KE, Wiggins J, Mead S, et al: Prevalence, characteristics, and survival of frontotemporal lobar degeneration syndromes. Neurology 86: 1736-1743, 2016.

10. Respondek G, Stamelou M, Kurz C, Ferguson LW, Rajput A Chiu WC, van Swieten JC, Troakes C, Al Sarraj S, Glepi E, et al: The phenotypic spectrum of progressive supranuclear palsy: A retrospective multicenter study of 100 definite cases. Mov Disord 29: 1758-1766, 2014.

11. Magdalinou NK, Paterson RW, Schott JM, Fox NC, Mummery C, Blennow K, Bhatia K, Morris HR, Giunti P, Warner TT, et al: A panel of nine cerebrospinal fluid biomarkers may identify patients with atypical parkinsonian syndromes. J Neurol Neurosurg Psychiatry 86: 1240-1247, 2015.

12. Smith PY, Delay C, Girard J, Papon MA, Planel E, Sergeant N, Buée L and Hébert SS: MicroRNA-132 loss is associated with tau exon 10 inclusion in progressive supranuclear palsy. Hum Mol Genet 20: 4016-4024, 2011.

13. Tatura R, Buchholz M, Dickson DW, van Swieten J, McLean C, Höglinger $\mathrm{G}$ and Müller U: MicroRNA profiling: Increased expression of miR-147a and miR-518e in progressive supranuclear palsy (PSP). Neurogenetics 17: 165-171, 2016.

14. Höglinger GU, Respondek G, Stamelou M, Kurz C, Josephs KA, Lang AE, Mollenhauer B, Müller U, Nilsson C, Whitwell JL, et al: Clinical diagnosis of progressive supranuclear palsy: The movement disorder society criteria. Mov Disord 32: 853-864, 2017.

15. Golbe LI and Ohman-Strickland PA: A clinical rating scale for progressive supranuclear palsy. Brain 130: 1552-1565, 2007.

16. Folstein MF, Folstein SE and McHugh PR: 'Mini-mental state': A practical method for grading the cognitive state of patients for the clinician. J Psychiatr Res 12: 189-198, 1975.

17. Dubois B, Slachevsky A, Litvan I and Pillon B: The FAB: A Frontal assessment battery at bedside. Neurology 55: 1621-1626, 2000.

18. Nasreddine ZS, Phillips NA, Bédirian V, Charbonneau S, Whitehead V, Collin I, Cummings JL and Chertkow H: The montreal cognitive assessment, MoCA: A brief screening tool for mild cognitive impairment. J Am Geriatr Soc 53: 695-699, 2005.

19. Quattrone A, Morelli M, Williams DR, Vescio B, Arabia G, Nigro S Nicoletti G, Salsone M, Novellino F, Nisticò R, et al: MR parkinsonism index predicts vertical supranuclear gaze palsy in patients with PSP-parkinsonism. Neurology 87: 1266-1273, 2016.

20. Quattrone A, Morelli M, Nigro S, Quattrone A, Vescio B, Arabia G Nicoletti G, Nisticò R, Salsone M, Novellino F, et al: A new MR imaging index for differentiation of progressive supranuclear palsy-parkinsonism from Parkinson's disease. Parkinsonism Relat Disord 54: 3-8, 2018.

21. Edgar R, Domrachev M and Lash AE: Gene expression omnibus: NCBI gene expression and hybridization array data repository. Nucleic Acids Res 30: 207-210, 2002

22. Conte I, Hadfield KD, Barbato S, Pizzo M, Bhat RS, Carissimo A Karali M, Porter LF, Urquhart J, Hateley S, et al: miR-204 is responsible for inherited retinal dystrophy associated with ocular coloboma. Proc Natl Acad Sci USA 112: E3236-E3245, 2015.

23. Gattolliat CH, Thomas L, Ciafrè SA, Meurice G, Le Teuff G, Job B, Richon C, Combaret V, Dessen P, Valteau-Couanet D, et al: Expression of miR-487b and miR-410 encoded by $14 \mathrm{q} 32.31$ locus is a prognostic marker in neuroblastoma. Br J Cancer 105: 1352-1361, 2011.

24. Liu X, He F, Pang R, Zhao D, Qiu W, Shan K, Zhang J, Lu Y, Li Y and Wang Y: Interleukin-17 (IL-17)-induced MicroRNA 873 (miR-873) contributes to the pathogenesis of experimental autoimmune encephalomyelitis by targeting A20 Ubiquitin-editing enzyme. J Biol Chem 289: 28971-28986, 2014.

25. Eiring AM, Harb JG, Neviani P, Garton C, Oaks JJ, Spizzo R, Liu S, Schwind S, Santhanam R, Hickey CJ, et al: miR-328 Functions as an RNA decoy to modulate hnRNP E2 Regulation of mRNA translation in leukemic blasts NIH Public Access. Cell 140: 652-665, 2010.

26. Schneider R, McKeever P, Kim TH, Graff C, van Swieten JC, Karydas A, Boxer A, Rosen H, Miller BL, Laforce R Jr, et al: Downregulation of exosomal miR-204-5p and miR-632 as a biomarker for FTD: A GENFI study. J Neurol Neurosurg Psychiatry 89: 851-858, 2018.

27. Starhof C, Hejl AM, Heegaard NHH, Carlsen AL, Burton M, Lilje B and Winge K: The biomarker potential of cell-free microRNA from cerebrospinal fluid in Parkinsonian syndromes. Mov Disord 34: 246-254, 2019.
28. Song S, Fajol A, Tu X, Ren B and Shi S: miR-204 suppresses the development and progression of human glioblastoma by targeting ATF2. Oncotarget 7: 70058-70065, 2016

29. Banaei-Esfahani A, Moazzeni H, Nosar PN, Amin S, Arefian E, Soleimani M, Yazdani S and Elahi E: MicroRNAs that target RGS5. Iran J Basic Med Sci 18: 108-114, 2015.

30. Pan BF, Gao C, Ren SX, Wang YB, Sun HP and Zhou MY: Regulation of PP2Cm expression by miRNA-204/211 and miRNA-22 in mouse and human cells. Acta Pharmacol Sin 36: 1480-1486, 2015.

31. Wang L, Tian H, Yuan J, Wu H, Wu J and Zhu X: CONSORT: Sam68 is directly regulated by miR-204 and promotes the self-renewal potential of breast cancer cells by activating the wnt/beta-catenin signaling pathway. Medicine (Baltimore) 94: e2228, 2015.

32. Yang $Y$ and Liang C: MicroRNAs: An emerging player in autophagy. ScienceOpen Res: Dec 22, 2015 (Epub ahead of print). doi: 10.14293/S2199-1006.1.SOR-LIFE.A181CU.v1.

33. Zhang S, Gao L, Thakur A, Shi P, Liu F, Feng J, Wang T, Liang Y, Liu JJ, Chen M and Ren H: MiRNA-204 suppresses human non-small cell lung cancer by targeting ATF2. Tumor Biol 37: $11177-11186,2016$

34. Li N, Guo X, Liu L, Wang L and Cheng R: Molecular mechanism of miR-204 regulates proliferation, apoptosis and autophagy of cervical cancer cells by targeting ATF2. Artif Cells, Nanomed Biotechnol 47: 2529-2535, 2019.

35. Katsuragi Y, Ichimura Y and Komatsu M: P62/SQSTM1 functions as a signaling hub and an autophagy adaptor. FEBS J 282: 4672-4678, 2015

36. Chi SW, Zang JB, Mele A and Darnell RB: Argonaute HITS-CLIP decodes microRNA-mRNA interaction maps. Nature 460: 479-486, 2009.

37. Weber A, Schwarz SC, Tost J, Trümbach D, Winter P, Busato F, Tacik P, Windhorst AC, Fagny M, Arzberger T, et al: Epigenome-wide DNA methylation profiling in progressive supranuclear palsy reveals major changes at DLX1. Nat Commun 9: 2929, 2018

38. Hafner M, Landthaler M, Burger L, Khorshid M, Hausser J, Berninger P, Rothballer A, Ascano M Jr, Jungkamp AC, Munschauer $\mathrm{M}$, et al: Transcriptome-wide identification of RNA-binding protein and microRNA target sites by PAR-CLIP. Cell 141: 129-141, 2010.

39. Xue Y, Ouyang K, Huang J, Zhou Y, Ouyang H, Li H, Wang G, Wu Q, Wei C, Bi Y, et al: Direct conversion of fibroblasts to neurons by reprogramming PTB-regulated microRNA circuits. Cell 152: $82-96,2013$

40. Krell J, Stebbing J, Carissimi C, Dabrowska AF, de Giorgio A, Frampton AE, Harding V, Fulci V, Macino G, Colombo T and Castellano L: TP53 regulates miRNA association with AGO2 to remodel the miRNA-mRNA interaction network. Genome Res 26: 331-341, 2016.

41. Obara Y, Sato H, Nakayama T, Kato T and Ishii K: Midnolin is a confirmed genetic risk factor for Parkinson's disease. Ann Clin Transl Neurol 6: 2205-2211,2019.

42. Obara Y, Imai T, Sato H, Takeda Y, Kato T and Ishii K: Midnolin is a novel regulator of parkin expression and is associated with Parkinson's disease. Sci Rep 7: 5885, 2017.

43. Karginov FV and Hannon GJ: Remodeling of Ago2-mRNA interactions upon cellular stress reflects miRNA complementarity and correlates with altered translation rates. Genes Dev 27: 1624-1632, 2013.

44. Spengler RM, Zhang X, Cheng C, McLendon JM, Skeie JM, Johnson FL, Davidson BL and Boudreau RL: Elucidation of transcriptome-wide microRNA binding sites in human cardiac tissues by Ago2 HITS-CLIP. Nucleic Acids Res 44: 7120-7131, 2016.

45. Ding X, Barodia SK, Ma L and Goldberg MS: Fbxl18 targets LRRK2 for proteasomal degradation and attenuates cell toxicity. Neurobiol Dis 98: 122-136, 2017

46. Upadhyay A, Amanullah A, Chhangani D, Mishra R, Prasad A and Mishra A: Mahogunin ring finger-1 (MGRN1), a multifaceted ubiquitin ligase: Recent unraveling of neurobiological mechanisms. Mol Neurobiol 53: 4484-4496, 2016.

47. Ramaswamy P, Christopher R, Pal PK and Yadav R: MicroRNAs to differentiate Parkinsonian disorders: Advances in biomarkers and therapeutics. J Neurol Sci 394: 26-37, 2018.

This work is licensed under a Creative Commons

Attribution-NonCommercial-NoDerivatives 4.0 International (CC BY-NC-ND 4.0) License. 